\section{Not entirely a backwater}

SIR - Barbara J. Culliton tells us that Texas, now honoured with two Nobel prizewinners, used to be a scientific backwater (Nature 357, 623; 1992), but that does not fit with history. The zoology department of the University of Texas at Austin, for example, has been for decades a stronghold of Drosophila and population genetics.

Hermann J. Muller made his outstanding experimental contributions to the study of heredity in plants and animals there, culminating in 1927 with reproducing mutations and producing novel ones by irradiation, an achievement for which he was awarded the Nobel prize in 1946, nearly 40 years ahead of Michael Brown and Joseph Goldstein.

Before and after the Second World War, the same department was a favourite for postdocs and other working visits by population geneticists. It produced quite a few of its own. In 1966, simultaneously with the teams of Harry Harris in London and R. C. Lewontin in Chicago, W. S. Stone's group in Austin discovered, through protein gel electrophoresis, the high level of gene heterozygosity in natural populations, contrary to Muller's contention (Proc. natn. Acad. Sci. U.S.A. 56, 119-125; 1966).

Through the early 1970 s, M. R. Wheeler at Austin put out the widely read Studies in Genetics series. Wheeler maintained the largest, healthiest and most varied existing collection of live fruitflies, meeting requests for stocks worldwide, and Stone was a key participant in a project for investigating Hawaiian drosophilids which, in the mid1960 s, received half a million dollars, an enormous amount for a research grant in biology at the time. Among Drosophila workers, the Stone group was famous for unparalleled preparations and pictures of giant salivary gland chromosomes.

The idea in the United States that anywhere but the east or west coast is a backwater strikes an all too familiar chord here in France, but several scientists from places other than Paris have nevertheless won Nobel prizes.

\section{Georges Pasteur}

Ecole Pratique des Haute Etudes,

Place Eugène Bataillon,

34095 Montpellier 05, France

\section{Italian science}

SIR - As members of the Science Committee of the Italian Space Agency (ASI) we feel obliged to rectify some of the statements made by Alison Abbott (Nature 357, 351; 1992).
It is obvious that the committee as a whole would welcome an increase of funds for basic science. The position expressed by the research minister in this respect is particularly important. It should be stressed, however, that "basic science" in ASI includes disciplines that are considered "applications" in the European Space Agency. ASI, at various levels, will have to engage in a careful discussion on how to apply the directives of the ministry.

We are concerned about the present functioning of the agency but we strongly feel that tilting at windmills will lead nowhere. So we have not changed our minds (as claimed in the article) and most of the members remain extremely critical of Professor Remo Ruffini's attitude, his personalistic approach and his poor management of the committees even in trivial matters.

It is probably unprecedented in science for an elected officer not to resign after a majority vote of no-confidence. We agree instead with Professor Giovanni Bignami that the internal mechnisms of the committee and of ASI itself (including the refereeing process) will require a careful, responsible re-examination.

Franco Pacini (Osservatorio Astrofisico de Arcetri, Largo Enrico Fermi 5, 50125

Florence, Italy); Giuliano Boella (University of Milan); Ezio Bussoletti (Naval University of Naples); Marino Dobrowolny (Institute of Interplanetary Plasma, Frascati); Sergio Vetrella (University of Naples)

\section{The biter bit}

SIR - A. P. R. Cooper ${ }^{1}$ accuses Daedalus $^{2}$ of not having done his homework. It seems that Cooper has not done his either. The scheme proposed by Daedalus is not the well-known 'Lempel-Ziv-Welch' (LZW) algorithm ${ }^{3}$ for data compression. The LZW compression algorithm builds strings ('words') from input data, generating a good dictionary from most data with no previous knowledge of their contents. It is a variation of an algorithm by Lempel and $\mathrm{Ziv}^{4}$. In fact, a person knowing the current data-compression techniques would be tempted to divide Daedalus's scheme into two parts:

(1) The idea of representing each word (or phrase) in a text by a look-up, or pointer, into a static dictionary and (2) the idea of representing each pointer with a number of bits according to the probability of occurrence.

Part (1) has not got a name, as it is regarded as 'immediately obvious' and no one has (yet) claimed credit, but it could, with a bit of tolerance, be called a variation of the 'Lempel-Ziv' data compression algorithm. Part (2) is also a well-known technique, but is generally used only on pure character data. The idea of using fewer bits for more common characters was proposed in the mid-1960s, and is currently taught at university level as 'Huffman-encoding'. This is not done in quite the way Daedalus proposed, as his algorithm cannot identify the length of the code for each word. To avoid this problem, Huffmanencoding is constructed so that no code introduces another code. An example can be given using Daedalus's scheme: assume that the, $a$ and an are the most common words in Nature, in that order. Then the would be represented by binary $0, a$ would be represented by binary 1 , and an would be represented by binary 10. If the program encounters the bits 10 , it cannot tell whether this is supposed to mean 'a the' or 'an'.

\section{Elvind Eklund}

Granveien 30 ,

N-1430 Ås, Norway
1. Nature 358, 365 (1992)
2. Nature 358, 22 (1992).
3. Welch. T A Computer (June 1984)
4. Ziv, J. \& Lempel, A. I.E.E.E. Transactions on Information Theory (May 1977)

\section{The reviewer reviewed}

SIR - Book reviews, as is well-known, say as much about reviewer as reviewed; in some cases they may even serve as a form of therapy. But there is a limit. and Stuart Sutherland (Nature 357, 550; 1992) with his dismissive aside on the "crackpots" J. B. S. Haldane and J. D. Bernal, is, I suggest, the wrong side of it.

Bernal and Haldane, respectively primarily crystallographer and geneticist, but both indisputably polymaths, were not merely innovative experimental and theoretical researchers within the laboratory; both were inspired visionaries and writers about the goals and purposes of science in its widest social context outside the laboratory. No one who attended the recent centenary meeting for Haldane at the Science Museum, or who rereads today Bernal's Science in History or The Social Functions of Scien$c e$, is likely to be in any doubt about this.

Although Bernal and Haldane are likely to be remembered long after Sutherland's sniping - and indeed the book he was ostensibly reviewing - are forgotten, it is a pity that Nature's pages should be thus occupied.

\section{Steven Rose}

Department of Biology,

Open University

Walton Hall,

Milton Keynes MK7 6AA, UK

NATURE . VOL 359 - 3 SEPTEMBER 1992 